\title{
Síndrome de Down y hernia de Morgagni en lactante
}

\author{
Down syndrome and Morgagni hernia in infant
}

\author{
Diana López-Gulfo,* Iván de la Peña-Sanabria, ${ }^{\ddagger}$ Jorge Duván Gulfo-Revolledo, ${ }^{\S}$ \\ Manuel Sánchez-Peralta, "José Pineda-Marriagall \\ * Universidad Autónoma de Bucaramanga, residente de Pediatría, Universidad del Sinú-Seccional Cartagena; \\ ‡ Universidad del Norte; Fundación Hospital de la Misericordia, Clínica de Occidente; § Corporación Universitaria \\ Rafael Núñez. "Universidad Surcolombiana; " Universidad Libre de Barranquilla, Colombia.
}

\begin{abstract}
RESUMEN
Introducción: La hernia de Morgagni es un defecto congénito que se presenta en más de $30 \%$ de los pacientes con síndrome de Down. Presentación del caso: Se trata de una paciente de 13 meses, quien ingresa con sintomatología respiratoria, sospechando inicialmente neumonía adquirida en comunidad; sin embargo, posteriormente se corrobora por Rx tórax que se trata de una hernia de Morgagni. Se realizó cirugía laparoscópica para su corrección, teniendo buena evolución postoperatoria. Conclusión: En niños con síndrome de Down con sintomatología respiratoria persistente se debe sospechar de hernia de Morgagni, ya que es una malformación congénita frecuente en este grupo de pacientes.
\end{abstract}

Palabras clave: Lactante, síndrome de Down, hernia de Morgagni, hernia diafragmática congénita.

\section{INTRODUCCIÓN}

La hernia de Morgagni (HM) fue descrita por primera vez por Giovanni Morgagni en $1761 .^{1-3}$ Es un defecto congénito poco frecuente,,$^{1,4-6}$ con una incidencia de 1:2,500-5,000 nacidos vivos y comprende de $3-5 \%$ de todas las hernias diafragmáticas congénitas, ${ }^{2,3}$ con una alta mortalidad neonatal aproximadamente de $10-30 \% .7,8$

LA HM se desarrolla debido a una fusión o desarrollo deficiente del diafragma y los arcos costales, ${ }^{2} \mathrm{y}$

\begin{abstract}
Introduction: Morgagni hernia is a birth defect that occurs in more than $30 \%$ of patients with Down syndrome. Case presentation: This is a thirteen-month-old female patient, who was admitted with respiratory symptoms, initially suspecting community-acquired pneumonia; however, a later chest $X$-ray confirmed that it was a Morgagni hernia. Laparoscopic surgery was performed with good postoperative evolution. Conclusion: In children with Down syndrome with persistent respiratory symptoms, Morgagni hernia should be suspected, since it is a common congenital malformation in these patients.
\end{abstract}

Keywords: Infant, Down syndrome, Morgagni hernia, congenital diaphragmatic hernia.

se caracteriza por una hernia anterior de los órganos abdominales hacia la cavidad torácica a través de un defecto diafragmático llamado agujero de Morgagni, a través del que puede protruir el epiplón, colon, estómago, hígado e intestino delgado., ${ }^{2,3,5,6} \mathrm{El}$ defecto está localizado en la porción anteromedial del diafragma, entre el xifoides del esternón y las fibras del margen costal. $^{9}$

El diagnóstico se puede realizar prenatalmente por ultrasonido o resonancia magnética; sin embargo,

Correspondencia: Jorge Duván Gulfo-Revolledo, E-mail: jgulfor10@gmail.com

Citar como: López-Gulfo D, De la Peña-Sanabria I, Gulfo-Revolledo JD, Sánchez-Peralta M, Pineda-Marriaga J. Síndrome de Down y hernia de Morgagni en lactante. Rev Mex Pediatr. 2021; 88(5): 189-191. https://dx.doi.org/10.35366/103898 
de $20-30 \%$ se detectan por primera vez después del nacimiento. ${ }^{1,10} \mathrm{El}$ principal estudio diagnóstico es la radiografía de tórax ${ }^{3}$ pero también son de utilidad las Rx de abdomen, y la tomografía axial computarizada de tórax. ${ }^{4,6}$ Dentro de los diagnósticos diferenciales se incluyen a atelectasias, derrame pleural, y parálisis diafragmática. En general, el tratamiento es quirúrgico, mediante cirugía abierta (transabdominal o transtorácica) o por laparoscópica ${ }^{3,6,9}$

En este artículo exponemos el caso de una paciente lactante con síndrome de Down, quien ingresa con sintomatología respiratoria, con sospecha inicial de neumonía adquirida en comunidad, pero posteriormente se confirma HM.

\section{PRESENTACIÓN DEL CASO}

Paciente femenino de 13 meses, originaria y residente de Bogotá, Colombia. El abuelo paterno con diagnóstico de síndrome de Down. Ingresó en compañía de su madre, quien refirió un cuadro clínico de siete días de evolución caracterizado por tos productiva, no emetizante, no cianosante, acompañada de rinorrea hialina y fiebre de $38{ }^{\circ} \mathrm{C}$. Acudió a urgencias de un centro de salud de primer nivel de atención, donde la encuentran con desaturación y aumento de trabajo respiratorio, por lo que indican oxígeno suplementa-

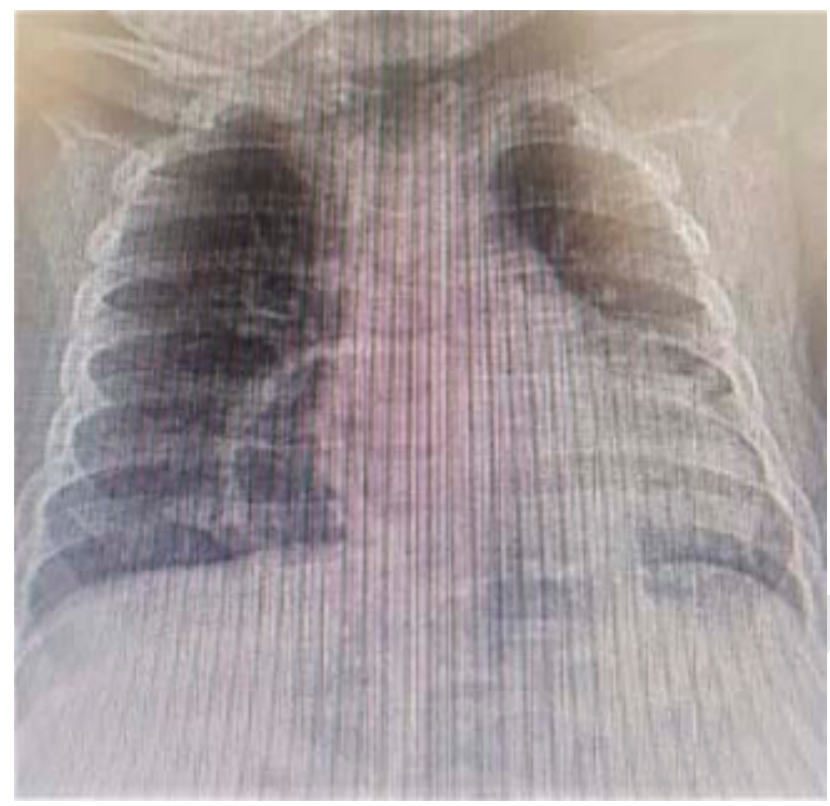

Figura 1: Radiografía de tórax anteroposterior que muestra imagen sugestiva de hernia diafragmática derecha.

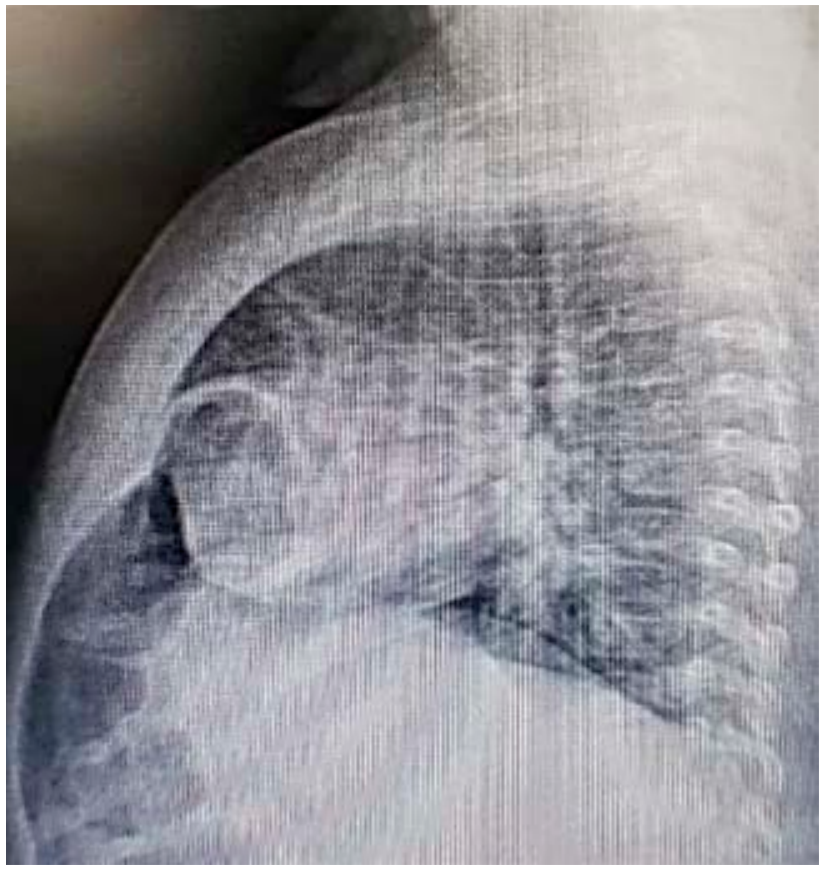

Figura 2: Radiografía de tórax lateral que evidencia asas intestinales en cavidad torácica.

rio. Se sospechó neumonía. En biometría hemática se evidencia leucocitosis con neutrofilia, así como anemia microcítica hipocrómica. En Rx de tórax se observó imagen redondeada radiolúcida, con borde radiopaco bien delimitado, lo cual se identificó como hernia diafragmática anterior. Se inició tratamiento con penicilina cristalina. Dado que la paciente presenta deterioro clínico, se decide trasladarla a un centro de salud de mayor complejidad.

En el examen físico a su ingreso a la institución especializada, se encontró en buenas condiciones generales, peso de $10.2 \mathrm{~kg}$, talla de $76 \mathrm{~cm}$, con estado nutricional normal (acorde a las curvas de crecimiento para niños con síndrome de Down), con dificultad respiratoria leve, desaturación que se corregía con oxigenoterapia a bajo flujo. En la exploración del tórax se detectó presencia de crépitos finos en ambas bases pulmonares. Al revisar la Rx de tórax se corrobora diagnóstico de hernia diafragmática, pero sin datos de neumonía.

Al segundo día de ingreso, el servicio de Cirugía Pediátrica confirma que la hernia diafragmática anterior (Figuras 1 y 2) es de tipo HM, y consideran manejo quirúrgico. Al tercer día se corrige por laparoscopía, sin complicaciones. La evolución postquirúrgica fue favorable. 


\section{DISCUSIÓN}

La hernia diafragmática congénita se denomina como de Morgagni, subesternocostal o retrocostoxifoidea cuando se encuentra al lado derecho del tórax, ${ }^{6}$ existe cierta discrepancia acerca de la frecuencia y el tipo de presentación, siendo menos común el tipo bilateral (21\%) que el unilateral (57\%); ${ }^{2}$ el caso presentado se trató de una unilateral derecha. En un estudio realizado por Escarcega y colaboradores se encontró que hubo un número igual de hernias del lado derecho e izquierdo, a pesar de que las hernias del lado izquierdo son históricamente menos frecuentes, debido a las inserciones pericárdicas en el diafragma que confieren protección y apoyo ${ }^{2}$ sin embargo, en un estudio en la ciudad de Medellín (Colombia) se encontró un predominio del lado izquierdo (83.3\%), con una frecuencia de 4.5 casos por año, entre 1999 y $2009 .{ }^{11}$

Uno de cada 733 nacidos vivos en los Estados Unidos de Norteamérica nace con síndrome de Down (SD). ${ }^{12}$ Estos pacientes tienen mayor riesgo de presentar anomalías congénitas; en particular las hernias diafragmáticas ocurren en más de $30 \%{ }^{2,6}$ En una cohorte de niños con hernia diafragmática realizada en el año 2018 se encontró una prevalencia de $57.1 \%$ de SD. ${ }^{9}$

Los pacientes con SD pueden tener varios motivos de dificultad respiratoria, como infecciones respiratorias, cardiopatía congénita, reflujo gastroesofágico o dificultades para la deglución, lo cual debe incluirse como parte del diagnóstico diferencial de la HM. ${ }^{13}$ Se ha descrito que los pacientes con HM se presentan con síntomas respiratorios en $56.5 \%$, y 19\% con síntomas gastrointestinales; en raras ocasiones puede haber obstrucción intestinal, malrotación intestinal o vólvulo gástrico. ${ }^{6,9,10}$ La tos persistente fue la característica de presentación más común (78\%) en una serie realizada por Rattan y colegas, seguida de vómitos recurrentes en $37 \%$ y estreñimiento en $9 \%$. Se encontró dolor abdominal agudo en $18 \%$ de los niños mayores de cuatro años. ${ }^{14}$

\section{REFERENCIAS}

1. Karmazyn B, Shold AJ, Delaney LR, Brown BP, Marine MB, Jennings $S G$ et al. Ultrasound evaluation of right diaphragmatic eventration and hernia. Pediatr Radiol. 2019; 49(8): 1010-1017.
2. Escarcega P, Riquelme MA, Lopez S, González AD, Leon VY, Garcia LR et al. Multi-institution case series of pediatric patients with laparoscopic repair of Morgagni hernia. $J$ Laparoendosc $A d v$ Surg Tech A. 2018; 28(8): 1019-1022.

3. Lauriti G, Zani-Ruttenstock E, Catania VD, Antounians L, Lelli Chiesa P, Pierro A et al. Open versus laparoscopic approach for Morgagni's hernia in infants and children: a systematic review and meta-analysis. J Laparoendosc Adv Surg Tech A. 2018; 28(7): 888-893.

4. Resch B, Liziczai K, Reiterer F, Freidl T, Haim M, Urlesberger B. Respiratory syncytial virus associated hospitalizations in children with congenital diaphragmatic hernia. Pediatr Neonatol. 2018; 59(2): 184-188.

5. Arikan S, Dogan MB, Kocakusak A, Ersoz F, Sari S, Duzkoylu Y et al. Morgagni's hernia: analysis of 21 patients with our clinical experience in diagnosis and treatment. Indian J Surg. 2018; 80(3): 239-244.

6. Slepov O, Kurinnyi S, Ponomarenko O, Migur M. Congenital retrosternal hernias of Morgagni: Manifestation and treatment in children. Afr J Paediatr Surg AJPS. 2016; 13(2): 57-62.

7. Benoist G, Mokhtari M, Deschildre A, Khen-Dunlop N, Storme L, Benachi $A$ et al. Risk of readmission for wheezing during infancy in children with congenital diaphragmatic hernia. PloS One. 2016; 11(5): e0155556.

8. Kadir D, Lilja HE. Risk factors for postoperative mortality in congenital diaphragmatic hernia: a single-centre observational study. Pediatr Surg Int. 2017; 33(3): 317-323.

9. Esposito C, Escolino M, Varlet F, Saxena A, Irtan S, Philippe P et al. Technical standardization of laparoscopic repair of Morgagni diaphragmatic hernia in children: results of a multicentric survey on 43 patients. Surg Endosc. 2017; 31 (8): 3320-3325.

10. Tan Y-W, Banerjee D, Cross KM, De Coppi P, GOSH team, Blackburn SC et al. Morgagni hernia repair in children over two decades: Institutional experience, systematic review, and metaanalysis of 296 patients. J Pediatr Surg. 2018; 53(10): 1883-1889.

11. Herrera Toro MN, Arango Rave ME, Tamayo Pérez ME. Hernia diafragmática congénita. Experiencia en el Hospital Universitario San Vicente de Paúl, Medellín, Colombia, 1999-2009. latreia. 2012; 25(3): 210-218.

12. Cua CL, Haque U, Santoro S, Nicholson L, Backes CH. Differences in mortality characteristics in neonates with Down's syndrome. $J$ Perinatol. 2017; 37(4): 427-431.

13. Jetley NK, Al-Assiri AH, Al-Helal AS, Al-Bin Ali AM. Down's syndrome as a factor in the diagnosis, management, and outcome in patients of Morgagni hernia. J Pediatr Surg. 2011; 46(4): 636639.

14. Rattan KN, Singh J, Dalal P. Diagnostic challenges in latepresenting congenital diaphragmatic hernia: a 16-year experience from tertiary care centre in North India. Trop Doct. 2019; 49(2): 138-141.

Conflicto de intereses: Los autores declaran que no tienen. 